\title{
Effects of Uncorrelated and Correlated Noise on Image Information Content
}

\author{
Ram M. Narayanan ${ }^{\star}$, Sudhir K. Ponnappan ${ }^{\star}$, and Stephen E. Reichenbach ${ }^{\dagger}$ \\ ${ }^{\star}$ Department of Electrical Engineering and Center for Electro-Optics, University of Nebraska, \\ 209N Walter Scott Engineering Center, Lincoln, NE 68588-0511, USA \\ Tel: +1-402-472-5141 Fax: +1-402-472-4732 EMail: rnarayanan@unl.edu \\ ${ }^{\dagger}$ Department of Computer Science and Engineering, University of Nebraska, \\ 115 Ferguson Hall, Lincoln, NE 68588-0115, USA \\ Tel: +1-402-472-5007 Fax: +1-402-472-7767 EMail: reich@unl.edu
}

\begin{abstract}
The information content in remote sensing imagery depends upon various factors such as spatial and radiometric resolutions, radiometric contrast between different target types, and also the final application for which the imagery has been acquired. Our approach to quantifying image information content is based upon classification accuracy. As noise is added to the image, the classification accuracy reduces, thereby resulting in loss of "information". The relationship between the information content and the noise variance can be described by a negative exponential model. The model is seen to be applicable for relating the information content to noise variance for Landsat TM as well as multi-look and single-look SIR-C imagery. We observe that the relationship is independent of the type of noise (Gaussian, Rayleigh, or Gamma). However, the rate of information loss increases with the correlation distance in the case of spatially correlated noise. The rate of information loss also increases with the number of classes chosen for classifying the scene. The model is useful in deducing allowable signal-to-noise ratios (SNRs) for different sensor systems.
\end{abstract}

\section{INTRODUCTION}

Various textural measures are used to characterize the image information content. Previous work in this area have resulted in three different approaches for quantifying the image information content, primarily based on interpretability, mutual information, and entropy [1]-[3]. These approaches, although well-refined, are difficult to apply to all types of remote sensing imagery. In a majority of cases, the raw image acquired by the sensor is processed using various operations such as filtering, compression, enhancement, etc. In performing these operations, the analyst is attempting to maximize the information content in the image to fulfill the end objective. In addition to noise added inherently by a sensor, image processing techniques corrupt the image with noise. It may seem obvious that as the image is corrupted with noise, the information content reduces, but in remote sensing images there may not be as great a loss as we might expect. This can be attributed to the fact that, although the value of a pixel may change as a result of corruption due to noise, the same pixel may in most cases, be correctly classified. Thus, an understanding of the effects of noise on image quality and interpretability is important from the standpoint of an deducing an “optimal SNR" depending on the end application.

The following empirical model for information content was developed.

$$
I=\exp \left\{-k(V)^{n}\right\}
$$

In Eq.(1), $I$ is the image information content, $V$ is the variance of the noise added, $k$ and $n$ are best-fit sensor specific constants.

The information content thus obtained can be considered to represent an utility index ranging from 0 to 1 . The model was developed based on the assumption that the reference image or "ground-truth" is the classified image with no noise added. Maximum achievable information is that value of $I$ obtained from the "ground-truth" image with the number of classes remaining constant. Our model formulation is intuitively appealing, since for $V=0, I=1$, i.e., all the information is preserved, while for increasing values of $V$, there is a decrease in the information about the textural aspects of the scene.

\section{PROCEDURE FOR ANALYSIS OF DATA}

Both TM and SIR-C imagery were analyzed. The method of analysis and consisted of the following steps.

(1) Choose the bands in the original image to be used for generating the classified image, (2) Perform an unsupervised classification of the chosen bands of the original image, by using the K-means approach for number of classes $\left(N_{c}=\right.$ $5,10,15)$, (3) Obtain the "ground-truth" or reference image from classification using the original bands, for each value of $N_{c}$, (4) For each band, corrupt the image by adding zero mean noise samples with increasing variance and reclassify the image, (5) Compare the degraded image with the reference image to obtain the actual information content $\hat{I}$, (6) Plot $\hat{I}$ obtained versus $V$, (7) Determine the best-fit constants $k$ and $n$, (8) Substitute the values of $k$ and $n$ in the model to generate a plot of $I$ versus $V$ for values of $V$ ranging from 0 to 2000, (9) Compare the plots from steps (6) and (7) to determine the accuracy of the model.

The same procedure is repeated for noise samples with different distributions and spatially correlated characteristics. The spatial correlation analysis was performed for circular correlation lengths of 20,50,100. Since we are investigating the interpretability of classified remote sensing images, we need to compare the "ground-truth" image with the classified degraded image to ascertain the latter's information content. 
The pixel value in the noise corrupted image will change depending upon the value of the noise sample that gets added to the pixel in the original image. A loss in information would occur whenever a pixel in the classified degraded image is misclassified in comparison to the same pixel in the "groundtruth" image. Hence classification accuracy would be a good measure of information content, $I$. A value of $I=1$ indicates that the classified degraded image is identical in interpretability to the "ground-truth" image, while $I=0$ indicates that the classified degraded image has no interpretability value for the scene that it represents.

\section{SITE DESCRIPTION}

The chosen scene shown in the Figure 1, includes parts of Nebraska, Iowa and Missouri. This sub-scene was extracted from Landsat Thematic Mapper (TM) scene acquired on August 26, 1991 with central latitude and longitude of $40.6^{\circ} \mathrm{N}$ and $95.7^{\circ} \mathrm{W}$ respectively. TM collects radiance data in seven bands between 0.45 and $12.5 \mu \mathrm{m}$. All six bands of data having a spatial resolution of $30 \mathrm{~m}$, except Band- 6 which has a spatial resolution of $120 \mathrm{~m}$, were used for analysis. The image is predominantly homogeneous with separable classes like soil, water, lowlands, rock and trees. The small town in the image is Hamburg and the only visible road stretching along is Highway 29. The image shown in Figure 1 is a false color composite image obtained using Bands 2, 3, and 4.

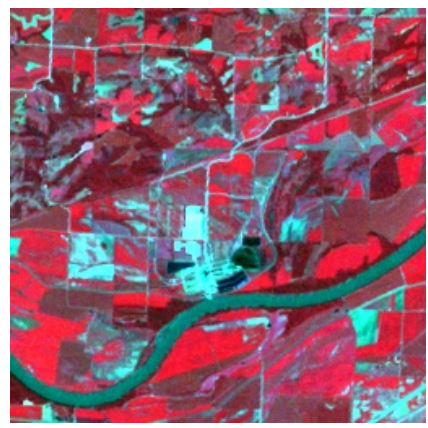

Fig. 1. The color composite image using TM Bands 2, 3 and 4, of Hamburg, Iowa, and surrounding area.

\section{INFORMATION ANALYSIS \& MODEL EFFICIENCY}

Six TM bands (Bands 1-5 and 7) were used to obtain the Kmeans classified images for each value of $V$. For each value of $V, \hat{I}$ was calculated following which the best-fit values of $k$ and $n$ were obtained.

Figures 2 and 3 illustrate the visual effects of adding noise. It can be easily deduced that as the variance of the noise increases, there is greater misclassification in comparison to the reference image. When the variance of the noise added is relatively low, there is not much loss of information. This implies that the interpretability of the image decreases with the increasing noise variance. With $N_{c}=5$, the classified image with noise variance up to 200 cannot be visually distinguished from the reference image, as seen from Figures 2(a) and 2(c).

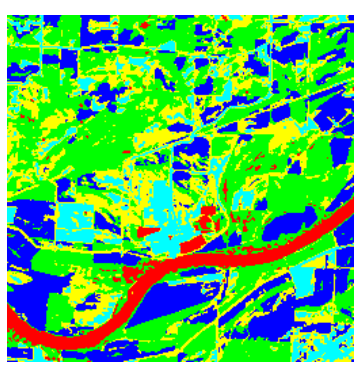

(a)

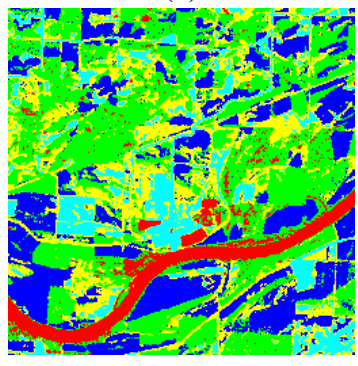

(c)

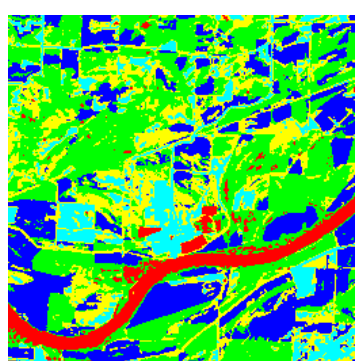

(b)

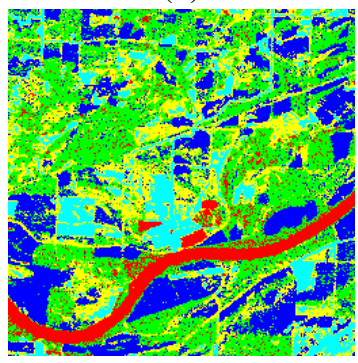

(d)
Fig. 2. Classified TM images with $N_{c}=5$, and (a) $V=0$, (b) $V=20$, (c) $V=200,($ d) $V=1000$.

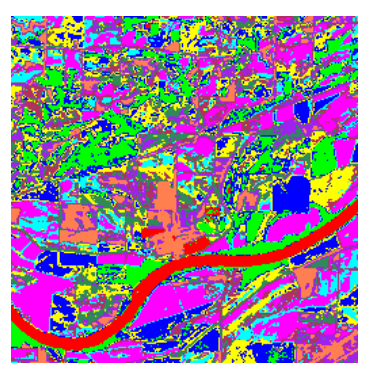

(a)

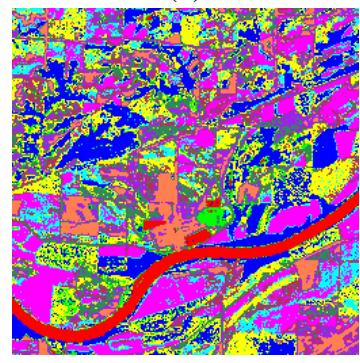

(c)

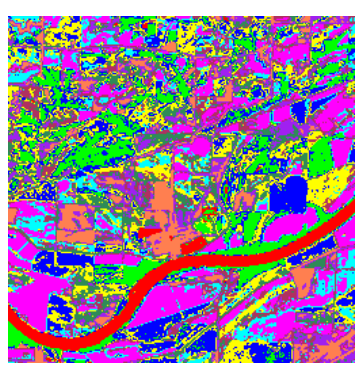

(b)

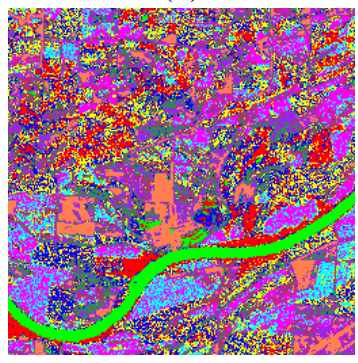

(d)
Fig. 3. Classified TM images with $N_{c}=10$, and (a) $V=0$, (b) $V=20$, (c) $V=200$, (d) $V=1000$.

As the number of classes increases, we can notice that the rate of fall of information also increases. This results from the creation of subclasses within the existing classes which increases the probability of misclassification of a pixel. This is also visually evident from the Figures 2 and 3. Comparing classified images for $N_{c}=5$ and $N_{c}=10$, it is visually evident that for the same noise variance, the classified image for $N_{c}=10$ has higher degradation. To check whether these ob- 
servations were mathematically consistent, plots relating the calculated information content, $I$, and noise variance, $V$, were studied for number of classes $N_{c}=\{5,10,15\}$.

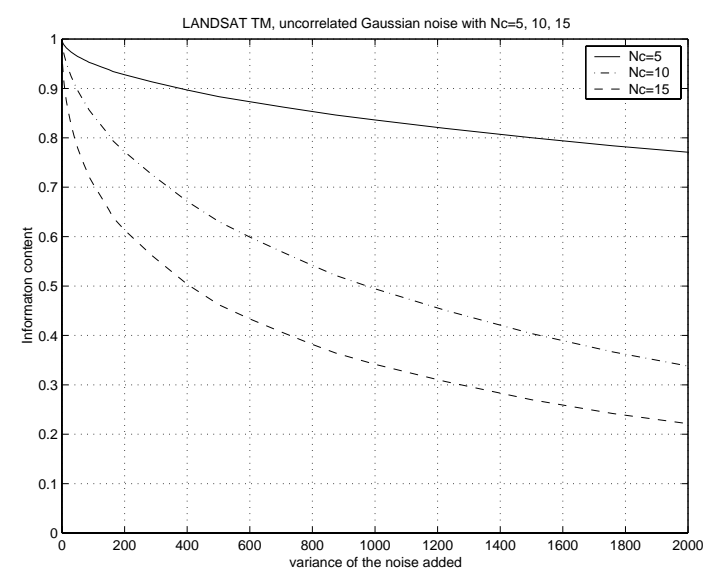

Fig. 4. Plots for TM image with $N_{c}=\{5,10,15\}$ are shown. Modeled information $I$ is shown as a function of $V$.

Figure 4 shows the plots for the TM scene, when the KMeans classifier was used with 5, 10 and 15 classes. On studying the plots for $I$, it can be seen that for number of classes $N_{c}$ equal to 5 , the image information content is about $83 \%$ for a noise variance of 1000 . Furthermore, as the number of classes in the scene increases, the rate of information loss also increases. For higher variances, the information content drops further. This supports our observations made from Figure 2, which shows minimal visual degradation for $V$ equal to 1000 .

A similar analysis was done for spatially correlated noise. Figure 5 shows the behavior of information content as a function of noise variance for different spatial correlation lengths. With increasing correlation length, there is a faster decay in information content, but this decay stabilizes after a certain correlation length.

\section{TABLE 1}

\section{$k$ AND $n$ VALUES FOR $N_{c}=\{5,10,15\}$, WITH CORRELATED AND UNCORRELATED NOISE FOR TM IMAGERY}

\begin{tabular}{|c|c|c|c|}
\hline $\begin{array}{c}\text { Correlation } \\
\text { Length }\end{array}$ & \multirow{2}{*}{$N_{c}$} & \multicolumn{2}{|c|}{ TM scene } \\
\cline { 3 - 4 } & $k$ & $n$ \\
\hline \hline 0 & 5 & 0.0043 & 0.5409 \\
\hline 0 & 10 & 0.0095 & 0.6229 \\
\hline 0 & 15 & 0.0365 & 0.6896 \\
\hline \hline 20 & 10 & 0.0175 & 0.6330 \\
\hline 50 & 10 & 0.0216 & 0.6134 \\
\hline 100 & 10 & 0.0240 & 0.6104 \\
\hline
\end{tabular}

Table 1 shows the values of $k$ and $n$ obtained for the models of $I$ with different $N_{c}$ and spatial correlation values. While $k$ increases with increasing values of $N_{c}, n$ increases with $N_{c}$ for the uncorrelated case but remains almost constant for a correlation length of 20. Our simulation experiments revealed that although the rate of information loss and $I$ are functions of both $k$ and $n$, the parameter $n$ has a greater influence on the rate of information loss than $k$.

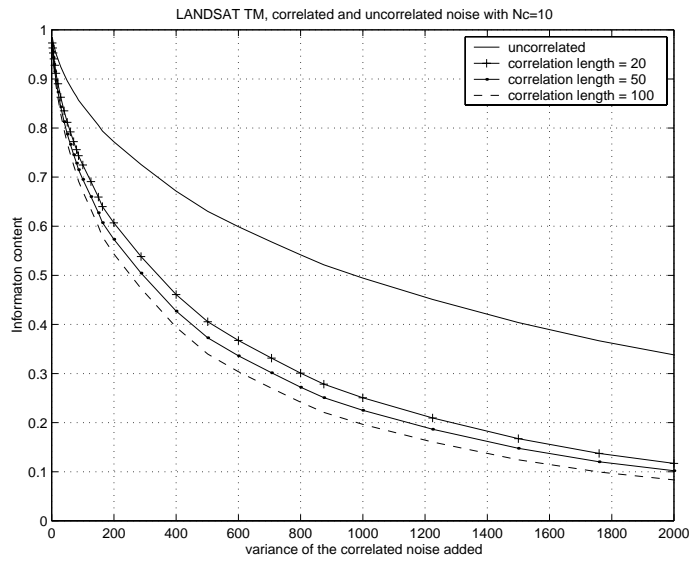

Fig. 5. Plots for TM image with $N_{c}=10$ and correlation length $=$ $\{0,20,50,100\}$ are shown. Modeled information $I$ is shown as a function of $V$.

\section{CONCLUSIONS}

This paper presents the effects of noise on information content of remote sensing imagery. Our formulation relates information content to accuracy of pixel classification. Simulations indicated that the behavior of information content is independent of the type of noise distribution. In addition, spatially correlated noise increases the rate of information decay. It is seen that to achieve an information content value of $80 \%$, maximum uncorrelated noise variances are 1482, 159 and 14 for number of classes equal to 5,10 , and 15 respectively. The model is thus useful in deducing allowable noise variances for different sensor systems based on the end application.

\section{ACKNOWLEDGMENT}

This work was supported by a NASA EPSCoR grant through the Nebraska Space Grant Consortium.

\section{REFERENCES}

[1] Moore, R.K.,"Tradeoff between picture element dimensions and noncoherent averaging in side-looking airborne radar," IEEE Transactions on Geoscience and Remote Sensing, vol. 15, No. 5, pp. 697-708, September 1979.

[2] Price, J.C., "Comparison of the information content of data from the Landsat-4 Thematic Mapper and the Multispectral Scanner," IEEE Transactions on Geoscience and Remote Sensing, vol. 22, No. 3, pp. 272-281, May 1984.

[3] Blacknell, D., and C.J. Oliver, "Information content of coherent images," Journal of Physics, vol. 26, No. 9, pp. 1364-1370, September 1993. 